Based on these results, POSCU services should be further analysed and considered in other areas and countries, promising a great beneficial impact on children and their families.

\section{THE OUTCOME OF A SUPERVISED, EXERCISE- PROGRAMME, FOR YOUNG PEOPLE WITH CEREBRAL PALSY: EFFECTS ON PHYSICAL ACTIVITY, FUNCTIONAL AND PARTICIPATION}

Natalie R Walsh, Sarah Rand, Hani F Ayyash, Michael Ogundele. UK

\subsection{6/bmjpo-2021-RCPCH. 146}

Background Young-people with Cerebral Palsy (CP) have been shown to have reduced physical fitness estimated at $15-28 \%$ less than healthy age matched children. As a result, they are at an increased risk of developing secondary health consequences due to a reduction in physical activity levels, lower cardiorespiratory capacity, and a decrease in muscle strength. There is limited research investigating the impact of a targeted exercise-programme for these young-people. The aim of this project was to conduct a service evaluation to explore the effects of a community-based, exercise-programme, for young people (age 9-13 years) with CP (GMFCS Levels-I-III).

Objectives

1. To design and implement a community-based exerciseprogramme for young-people with CP.

2. To investigate the impact on physical activity levels using daily step-count data and Physical Activity Questionnaire for Older Children (PAQ-C) scores.

3. To investigate the impact on functional-mobility using TimedUp-and-Go (TUG) times.

4. To investigate participation scores using the Child and Adolescent Scale of Participation (CASP) scores.

Methods Young people with CP were identified using inclusion and exclusion criteria from a single centre. Approvals were granted and consent was obtained from all participants and their parents/carers. A weekly, individualised, physiotherapyled, exercise-programme was completed at a large public leisure centre for six consecutive weeks. Outcome measures were completed to measure changes in physical activity, functionalmobility, and participation at pre-intervention, post-intervention and three-month follow-up.

Results Eight young-people with CP consented and four participants completed the intervention and outcomes. The mean daily step-count increased from post-intervention for three-participants (range -1914.06-2096.86 steps) and was higher on weekdays (range $6410(\mathrm{SD}= \pm 58.35)$ to 664.49 steps $(\mathrm{SD}=$ $\pm 1367.92)$ ) than weekend-days (range $461.35 \quad(\mathrm{SD}= \pm 67.84)$ to 5586.64 steps $(\mathrm{SD}= \pm 539.17))$.

The PAQ-C-scores increased for all participants post-intervention (within-participant-difference range (WPDR) 0.21 $(\mathrm{SD}= \pm 0.38)$ to $0.58(\mathrm{SD}= \pm 0.70))$ and varied at follow-up (WPDR $-0.46(\mathrm{SD}= \pm 0.72)$ to $0.69(\mathrm{SD}= \pm 0.80))$. The TUGtimes improved for two-participants post-intervention (WPDR $-24.29(\mathrm{SD}= \pm 6.13)$ to 1.28 seconds $(\mathrm{SD}= \pm 0.57))$ and was maintained at follow-up (WPDR $-23.70(\mathrm{SD}= \pm 6.22)$ to 1.17 seconds $(\mathrm{SD}= \pm 0.42))$. The CASP-scores improved for threeparticipants (range 2 to 11 ) and was maintained at follow-up (2 to 12 ).

Conclusions The aim of this project was to explore the feasibility and effects of a community-based exercise-group for young-people with CP. Overall the implementation and integration of the programme into the gym-environment was very successful. Attendance levels were $90 \%$ and all participants reported excellent subjective levels of enjoyment. The results of this project demonstrate preliminary, positive evidence for the effectiveness of the intervention on physical activity, functional-mobility, and participation measures in this small sample. The sample size and data collection were unfortunately affected by the Covid-19 pandemic. Therefore, additional data collection will be conducted in the future to further investigate the impact of the programme. It is clear that further studies are required to investigate outcome measures, to record the effects of interventions on physical activity, functional-mobility, and participation in young-people with CP. This preliminary study shows promise for the positive impact of community-based, exercise-programmes and should be considered as an additional option to facilitate young-people with $\mathrm{CP}$ to increase their participation, and to incorporate physical activity into their daily lives.

\section{EFFECT OF COVID-19 LOCKDOWN ON THE CONFIGURATION AND IMPLEMENTATION OF SERVICES FOR CHILDREN AND YOUNG PEOPLE WITH NEURODEVELOPMENTAL DISORDERS IN THE UK}

Michael Ogundele, Hani Ayyash. UK

\subsection{6/bmjpo-2021-RCPCH. 147}

Background The pandemic corona virus outbreak is having an impact on the lives of every member of the society with lockdown measures to limit the virus spreading through Schools shutting down, limited contact with family and friends, as well as social and leisure activities severely curtailed. This also necessitated unavoidable changes to various healthcare services provision worldwide.

Emotional, behavioural, Neurodevelopmental and Intellectual disorders (EBNDID) including ADHD, Autism, Epilepsy and Cerebral palsy affect up to $15 \%$ of the childhood population.

Objectives We aimed to identify pragmatic changes made to services provision for Children and Young people (CYP) with EBNDIDs and help disseminate examples of best practice among $\mathrm{CCH}$ Clinicans, while preparing for future pandemic lockdowns.

Methods We carried out a literature review about the impact of COVID-19 pandemic on healthcare services provided for children and adolescent with ADHD from several databases including Pubmed, PMC, CINAHL, Embase, PsycINFO and Ovid, with no limitations in terms of language and date of publication from inception to Dec 2020. This was supplemented by an online scoping survey among selected members of the George-Still Forum (National ADHD Network) run between June and July 2020.

Results 62 responses were obtained from the online $(62 \%$ rate). Several national and international professional bodies provided clinical guidance to clinicians about necessary service modifications to ensure that patients continue to have access to best evidence-based care despite the limitations imposed by the pandemic, but the awareness among frontline Paediatricians was limited. The clinical recommendations have centred around (i) expansion of various forms of remote and online services, (ii) Infection control strategies (iii) and staff redeployment to areas of higher clinical demands. $8 \%$ of the surveyed 
clinicians were not aware of any specific guidance for CYP with EBNDIDs.

Due to significant restriction to direct face-to-face clinician contacts and redeployment of staff from Community Child Health $(\mathrm{CCH})$ services, many core clinical activities became limited and most new referrals were kept on hold except for urgent services. $\mathrm{CCH}$ teams experienced significantly increased waiting lists. During the pandemic, many $\mathrm{CCH}$ services increased their telemedicine capacity exponentially. Telephone consultation was the commonest method of remote contact with patients (98\%).

Preliminary report has confirmed that the Black, Asian and Minority Ethnic staff and patient communities have been disproportionately affected by morbidity and mortality from COVID-19 infection. Parents and carers were encouraged to make more effective use of online resources including contacting NHS 111 or the GP and only if necessary to going the nearest Emergency Unit.

A wide array of online resources designed for CYP with EBNDID and their families including webinars, online videos, sleep tips, post-diagnosis support etc have become more popular.

Conclusions The pandemic has significantly impacted on routine service provision for new referral $s$ to $\mathrm{CCH}$ clinics and for the existing patients. Discontinuation of medical treatment necessitated by health service disruptions should be avoided during the future pandemic lockdowns to avoid serious detrimental effects of escalating challenging behaviour among CYP with EBNID, stressful parent-child and intra-sibling relationships and exacerbation of family disruptions.

\section{IMPROVING PATIENT FEEDBACK IN THE CHILDREN'S EMERGENCY DEPARTMENT - PART 1: IMPROVING THE QUALITY AND QUANTITY OF FEEDBACK GIVEN}

Tarn Stroud, Tarn Stroud, Ashvin Seetul, Patrick Robinson, Sally Melson, Anne Frampton. UK

\subsection{6/bmjpo-2021-RCPCH.148}

Background Listening to and acting upon feedback from patients and their parents and carers are vital dimensions of the delivery and design of excellent emergency care for sick and injured children. However, a nationwide staff survey in 2019 found that just $58.2 \%$ of staff in acute hospitals reported that feedback from service users was used to make informed decisions in their department. This study is the first part of a wider programme of work to increase patient and parent engagement in the Children's Emergency Department (CED).

Objectives To understand how patients and their parents/carers want to give feedback on their experiences in the CED, to inform the future development of a dedicated CED patient feedback tool.

Methods A questionnaire was designed in digital and paper formats with input from the hospital patient experience team. The questionnaire consisted of multiple choice and free-text questions. The paper questionnaire was offered to patients attending the CED over a six day period, and a poster advertising a Quick Response code enabling patients and parents to give feedback via a digital questionnaire was placed on the waiting room wall for fourteen days.

Results 51 responses (40 paper responses and 11 electronic) were obtained. $15.7 \%$ (8/51) of responses were completed by
Abstract 266 Table 1 How would patients/parents like to give feedback on their experiences in the Children's Emergency Department?

$\begin{array}{lll}\text { On a phone/mobile device } & 32 / 51 & 62.7 \% \\ \text { By email at home } & 18 / 51 & 35.3 \% \\ \text { Writing on paper } & 9 / 51 & 17.6 \% \\ \text { Via social media } & 4 / 51 & 7.8 \% \\ \text { Drawing on paper } & 3 / 51 & 5.9 \% \\ \text { Talking to someone } & 3 / 51 & 5.9 \%\end{array}$

patients themselves, and $84.3 \%(43 / 51)$ were completed an accompanying parent or guardian.

$54.9 \%(28 / 51)$ of responses related to pre-school age patients, $21.6 \%(11 / 51)$ related to primary school age patients, and $23.5 \%(12 / 51)$ related to secondary school aged children.

More than half of patients/parents (26/51) reported never having given feedback on hospital services in the past, and $33.3 \%(17 / 51)$ reported that they had previously given feedback. $15.7 \%$ (8/51) patients/parents were not sure whether they had given feedback before.

$35.2 \%(18 / 51)$ of respondents reported that they did not know how to give feedback on their experiences in CED.

When asked how they would like to feed back on their experiences in the Children's ED, a clear majority of respondents $(62.7 \%, 32 / 51)$ stated that they would like to give feedback using a phone/mobile device, and more than a third (18/ 51) wanted to use email. See table 1 below.

Conclusions This study indicates that there is scope to increase the number of patients and parents who give feedback on their experiences in the Children's Emergency Department, and clearly demonstrates the importance of access to digital methods and raising awareness to enable this. Combining this with the staff survey results in the second part of our study, we have designed a new tool for obtaining patient feedback. This tool will be piloted, and further work will be undertaken to evaluate whether improvements in the quantity and quality of patient feedback are achieved.

\section{CHALLENGES IN THE DIAGNOSIS OF COMMON NEONATAL CONDITIONS IN RESOURCE-LIMITED SETTINGS}

Aimee Staunton, Helen M Nabwera, Stephen J Allen. UK

\subsection{6/bmjpo-2021-RCPCH.149}

Background Accurate and timely diagnosis and management of common neonatal conditions is crucial for reducing preventable neonatal deaths. In low- and middle-income countries, there is sparse information on how neonatal diagnoses are made including use of clinical guidelines and access to appropriate laboratory and radiology services. An improved understanding of the diagnostic pathways for neonates in lowresource settings will help to inform future interventions and the development of best practice guidelines.

Objectives To identify challenges in neonatal diagnosis in resource-limited settings by describing diagnostic pathways for common neonatal conditions.

Methods Between August 2018 and April 2019, standard forms developed by the Neonatal Nutrition Network (NeoNu$\mathrm{Net}) *$ collaborators were completed by clinicians for all infants 